\title{
IRAS 08281-4850 and IRAS 14325-6428: two A-type post-AGB stars with s-process enrichment ${ }^{\star} \star \star \star$
}

\author{
M. Reyniers ${ }^{1, \star \star \star}$, G. C. Van de Steene ${ }^{2}$, P. A. M. van Hoof $^{2}$, and H. Van Winckel ${ }^{1}$ \\ ${ }^{1}$ Instituut voor Sterrenkunde, Departement Natuurkunde en Sterrenkunde, K.U.Leuven, Celestijnenlaan 200D, 3001 Leuven, \\ Belgium \\ 2 Koninklijke Sterrenwacht van België, Ringlaan 3, 1180 Brussel, Belgium
}

Received 4 April 2007 / Accepted 6 June 2007

\begin{abstract}
Aims. One of the puzzling findings in the study of the chemical evolution of (post-)AGB stars is why very similar stars (in terms of metallicity, spectral type, infrared properties, etc.) show a very different photospheric composition. We aim at extending the still limited sample of s-process enriched post-AGB stars, in order to obtain a statistically large enough sample that allows us to formulate conclusions concerning the 3rd dredge-up occurrence.

Methods. We selected two post-AGB stars on the basis of IR colours indicative of a past history of heavy mass loss: IRAS 082814850 and IRAS 14325-6428. They are cool sources in the locus of the Planetary Nebulae (PNe) in the IRAS colour-colour diagram. Abundances of both objects were derived for the first time on the basis of high-quality UVES and EMMI spectra, using a critically compiled line list with accurate $\log (g f)$ values, together with the latest Kurucz model atmospheres.

Results. Both objects have very similar spectroscopically defined effective temperatures of $7750-8000 \mathrm{~K}$. They are strongly carbon and s-process enriched, with a C/O ratio of 1.9 and 1.6, and an [1s/Fe] of +1.7 and +1.2 , for IRAS 08281-4850 and IRAS 14325-6428 respectively. Moreover, the spectral energy distributions (SEDs) point to heavy mass-loss during the preceding AGB phase.

Conclusions. IRAS 08281-4850 and IRAS 14325-6428 are prototypical post-AGB objects in the sense that they show strong post 3rd dredge-up chemical enrichments. The neutron irradiation has been extremely efficient, despite the only mild sub-solar metallicity. This is not conform with the recent chemical models. The existence of very similar post-AGB stars without any enrichment emphasizes our poor knowledge of the details of the AGB nucleosynthesis and dredge-up phenomena. We call for a very systematic chemical study of all cool sources in the PN region of the IRAS colour-colour diagram.
\end{abstract}

Key words. stars: AGB and post-AGB - stars: abundances - stars: carbon - stars: individual: IRAS 14325-6428 stars: individual: IRAS 08281-4850

\section{Introduction}

Post-AGB stars are key objects in the study of the dramatic chemical and morphological changes of objects on their ascent on the Asymptotic Giant Branch (AGB) and subsequent evolution. In this paper we report on our ongoing research to study the AGB chemical evolution by a systematic study of postAGB photospheres. Spectra of post-AGB stars are much easier to study than their AGB precursors for several reasons. First, their atmospheres do not show the large amplitude pulsations and the large mass loss rates that characterise AGB atmospheres. Second, their photospheres are hotter, so atomic transitions prevail in post-AGB spectra, while molecular transitions prevail in AGB star spectra. This allows us to quantify the chemical content of a very wide range of trace elements. Unfortunately, postAGB stars evolve on a very fast track and whether the current Galactic sample is representative is one of the most difficult issues when interpreting the results of single post-AGB stars in the broader context of stellar evolution. Therefore, extending the still limited sample of post-AGB stars is indispensable in order to attain a statistically large enough sample that allows us to

* Based on observations collected at the European Southern Observatory, Chile (programmes 70.D-0278(A) and 73.D-0241(A))

$\star \star$ Tables 4 et 5 are only available in electronic form at http: //www . aanda.org

$\star \star \star$ Postdoctoral fellow of the Fund for Scientific Research, Flanders. formulate conclusions concerning the (post-)AGB evolution in general.

During the past decennia, it has been realised that post-AGB stars are chemically much more diverse than previously thought. Binary objects tend to have a totally different photospheric composition than single objects, showing some degree of depletion of refractory elements in their photosphere (see e.g. Maas et al. 2005). The single objects, on their side, are also far from chemically homogeneous. Some objects are the most s-process enriched objects known to date (e.g. Reyniers et al. 2004, and references therein), while others are not enriched at all. This dichotomy is very strict, in the sense that mildly enhanced objects do not exist, except for a few rather atypical objects. Chemical evolutionary AGB models do predict that there is a minimum initial mass for the 3 rd dredge-up to occur, at around $1.4 M_{\odot}($ e.g. Straniero et al. 2003). Hence post-AGB stars without any helium burning products in their photosphere are theoretically expected. However a strict dichotomy is not expected, since a more gradual transition between non-enriched and enriched objects is predicted if the transition from an O-rich AGB to a C-rich AGB star occurs over many thermal pulses. Furthermore, the s-process enriched sub-class of post-AGB objects imposes another unsolved problem. These stars exhibit a large spread in s-process efficiency, as they do not obey the expected anti-correlation between metallicity and s-process efficiency (see Fig. 4 of Van Winckel 2003). In other words, examples exist of very similar post-AGB stars (in metallicity, spectral type, infrared excess, etc.), but with 


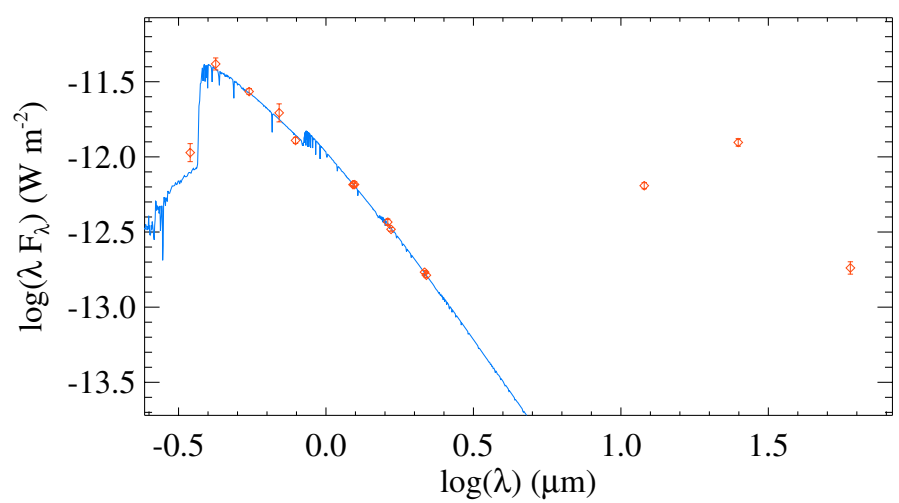

Fig. 1. The spectral energy distribution of IRAS 08281-4850.

Table 1. Basic parameters of the two objects discussed in this study.

\begin{tabular}{lrrr}
\hline \hline \multicolumn{1}{c}{ IRAS } & $08281-4850$ & $14325-6428$ \\
\hline Equatorial coord. & $\alpha_{2000}$ & 082940.552 & 143634.375 \\
& $\delta_{2000}$ & -490004.33 & -644131.09 \\
Galactic coord. & $l$ & 266.08 & 313.87 \\
& $b$ & -5.82 & -4.08 \\
Vis. magnitude & $V$ & 14.1 & 11.9 \\
Spectral Type & A9I & A8I \\
\hline Source SIMBAD, except magnitude and spectral type \\
(this work).
\end{tabular}

a totally different photospheric abundance pattern (see for example Fig. 1 in Van Winckel \& Reyniers 2001). This result dramatically illustrates that the $3 \mathrm{rd}$ dredge-up phenomenon is not yet fully understood.

In this paper, we selected two objects with far infrared colours typical of PNe from the IRAS point source catalogue. Apart from PNe, only post-AGB stars are typically found in this part of the colour-colour diagram (van Hoof et al. 1997). Furthermore, the selected objects were not detected in the radio continuum above a detection limit of $3 \mathrm{mJy}$ (Van de Steene \& Pottasch 1993, 1995). Hence they do not seem to have evolved to the PN stage as yet and are very likely post-AGB stars. We obtained JHKL images of the candidates with CASPIR on the 2.3-m telescope at Siding Spring Observatory in Australia in order to assure the correct identification of the IRAS counterparts and obtain accurate positions (Van de Steene et al. 2000). Two of these infrared selected post-AGB stars which are presented here (see Table 1) have counterparts in the USNO catalogue so that their visual magnitudes are also known. They were sufficiently bright to be observed in the optical at high resolution.

The paper is organised as follows: in the next section, we discuss the spectral energy distribution of the two objects, and quantify the total reddening towards the two sources. In Sect. 3 we briefly discuss the observation and reduction of the highresolution spectra, while in Sect. 4 we deal with the abundance analysis. Section 5 is a section devoted to the diffuse interstellar bands in the spectrum of IRAS 14325-6428. In the discussion (Sect. 6), we mainly focus on the s-process abundances. We end the paper with conclusions.

\section{Spectral energy distribution}

\subsection{Geneva photometry}

Images were obtained for both objects with the Swiss Euler telescope at La Silla (ESO) in 2005. The observing log is presented in Table 2. The objects were unresolved in all images. The
Table 2. Observational $\log$ for the images obtained at the Euler telescope at ESO, La Silla.

\begin{tabular}{rrrrr}
\hline \hline IRAS & Date & filter & exptime & airmass \\
\hline $08281-4850$ & $2005-10-07$ & UG & 300.2 & 1.473 \\
& & BG & 20.9 & 1.423 \\
& & VG & 150.5 & 1.408 \\
& & RG & 60.6 & 1.392 \\
& & IC & 98.9 & 1.351 \\
\hline $14325-6428$ & $2005-08-04$ & UG & 399.3 & 1.322 \\
& & BG & 299.5 & 1.311 \\
& & VG & 241.6 & 1.292 \\
\hline
\end{tabular}

images were bias subtracted and flatfielded in IRAF. The photometry package was used to do the aperture photometry. In case of crowded fields DAOPHOT was used to subtract the neighbours. One to three standard stars per band per star were available for the photometric calibration. The resulting magnitudes are presented in Table 3.

\subsection{Additional photometry}

We collected additional photometry from various surveys: the Tycho-2 Catalogue (Høg et al. 2000), the 3rd data release of DENIS (Epchtein et al. 1994), 2MASS (Skrutskie et al. 1997), MSX (Egan et al. 2003), and the IRAS point source catalogue (Beichmann 1985). We also obtained literature data from GarciaLario et al. (1997, hereafter GL97) and Van de Steene et al. (2000, hereafter VdS00). The data are presented in Table 3. Near-IR data of IRAS 08281-4850 were present in the 2nd release of the DENIS data set, but was deleted in the 3rd release. The data from the 2 nd release have not been retained in the analysis. In order to construct the spectral energy distribution (SED), we first converted the Tycho-2 magnitudes to the Johnson system using the formulas:

$B_{J}=B_{T}-0.240\left(B_{T}-V_{T}\right)$

$V_{J}=V_{T}-0.090\left(B_{T}-V_{T}\right)$

which were derived from Perryman et al. (1997). The Gunn-r magnitude from the Geneva system was converted to the Johnson system using the formula:

$R_{J}=r-0.43-0.15\left(B_{J}-V_{J}\right)$

(Kent 1985) where $B_{J}-V_{J}$ can be calculated from the Geneva photometry using Eqs. (12) and (13) from Harmanec \& Božić (2001). After that, the photometry was converted to $\lambda F_{\lambda}$ using the absolute flux calibrations given in Table 4. For the GL97 observations we adopt the Johnson photometric calibration, while for the VdS00 observations we adopt the MSSO photometric calibration. The resulting spectral energy distribution is shown in Table 5.

\subsection{Fitting the SED}

The SEDs of the post-AGB stars suffer from interstellar as well as circumstellar extinction. We assumed that this total extinction can be described by an $R_{V}=3.1$ galactic extinction curve as defined by Fitzpatrick (1999). We furthermore assumed that the intrinsic spectrum of the stars can be described by an Atlas stellar atmosphere model (Castelli \& Kurucz 2004) with the parameters given in Table 8 . We then used an iterative procedure to determine the amount of total extinction $A_{V}$ and the bolometric 
Table 3. Broadband photometry for IRAS 08281-4850 and IRAS 14325-6428. IRAS 14325-6428 is contained twice in the DENIS catalogue. The data sets do not agree within the error margins, which may point to variability of the source. Both data sets have been retained in the analysis.

\begin{tabular}{|c|c|c|c|c|}
\hline \multirow{2}{*}{ phot. band } & \multicolumn{2}{|c|}{$08281-4850$} & \multicolumn{2}{|c|}{$14325-6428$} \\
\hline & mag & $\Delta \mathrm{mag}$ & mag & $\Delta \mathrm{mag}$ \\
\hline Euler Geneva $U$ & 17.81 & 0.15 & 14.58 & 0.25 \\
\hline Euler Geneva $B$ & 14.76 & 0.10 & 12.08 & 0.10 \\
\hline Euler Geneva $V$ & 14.09 & 0.05 & 11.89 & 0.05 \\
\hline Euler Gunn- $r$ & 13.23 & 0.15 & & \\
\hline Euler $I_{C}$ & 12.20 & 0.05 & & \\
\hline Tycho-2 $B_{T}$ & & & 12.676 & 0.209 \\
\hline Tycho- $2 V_{T}$ & & & 12.065 & 0.169 \\
\hline DENIS Gunn- $i$ & & & 10.297 & 0.04 \\
\hline DENIS $J$ & & & 9.202 & 0.07 \\
\hline DENIS $K_{\mathrm{s}}$ & & & 8.445 & 0.09 \\
\hline DENIS Gunn- $i$ & & & 10.431 & 0.02 \\
\hline DENIS $J$ & & & 9.292 & 0.05 \\
\hline DENIS $K_{\mathrm{s}}$ & & & 8.604 & 0.05 \\
\hline 2MASS $J$ & 10.603 & 0.019 & 9.263 & 0.023 \\
\hline 2MASS $H$ & 10.124 & 0.023 & 8.860 & 0.025 \\
\hline 2 MASS $K_{\mathrm{s}}$ & 9.835 & 0.021 & 8.598 & 0.025 \\
\hline GL97 $J$ & 10.68 & 0.05 & & \\
\hline GL97 $H$ & 10.12 & 0.05 & & \\
\hline GL97 $K$ & 9.79 & 0.04 & & \\
\hline VdS00 J & & & 9.27 & 0.05 \\
\hline $\mathrm{VdS} 00 H$ & & & 8.81 & 0.05 \\
\hline VdS00 $K$ & & & 8.61 & 0.05 \\
\hline VdS00 $L$ & & & 8.27 & 0.10 \\
\hline & $\begin{array}{r}F_{v} \\
(\mathrm{Jy})\end{array}$ & $\begin{array}{l}\Delta F_{v} \\
(\mathrm{Jy})\end{array}$ & $\begin{array}{r}F_{v} \\
(\mathrm{Jy})\end{array}$ & $\begin{array}{l}\Delta F_{v} \\
(\mathrm{Jy})\end{array}$ \\
\hline MSX A 8.28 & & & 0.72 & 0.03 \\
\hline MSX C 12.13 & & & 3.35 & 0.18 \\
\hline MSX D 14.65 & & & 7.1 & 0.4 \\
\hline MSX E 21.34 & & & 17.7 & 1.1 \\
\hline IRAS 12 & 2.23 & 0.11 & 3.31 & 0.20 \\
\hline IRAS 25 & 9.83 & 0.59 & 30.6 & 1.2 \\
\hline IRAS 60 & 3.62 & 0.36 & 17.1 & 1.9 \\
\hline
\end{tabular}

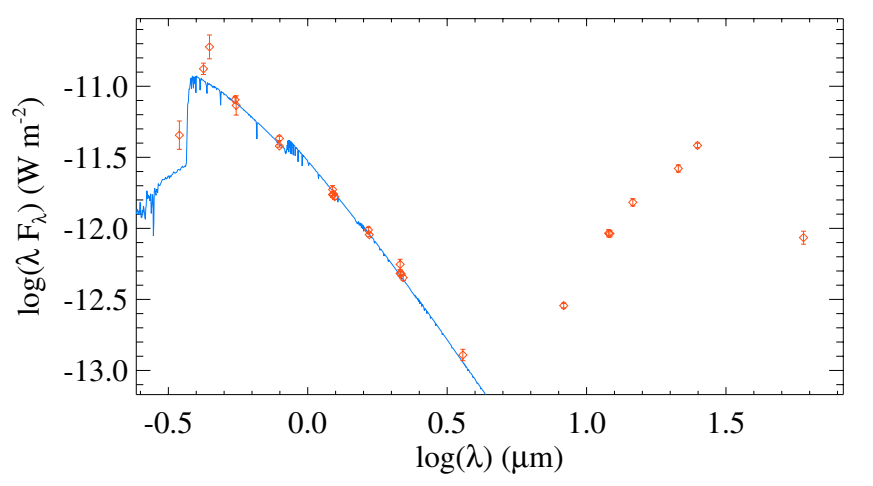

Fig. 2. The spectral energy distribution of IRAS 14325-6428.

flux $L /\left(4 \pi D^{2}\right)$ by minimizing the quadratic residuals of all observations between 0.4 and $4 \mu \mathrm{m}$. The results are given in Table 6 . Plots of the Kurucz stellar atmosphere models combined with the dereddened observed fluxes are shown in Figs. 1 and 2.

\section{High-resolution spectra: observation and reduction}

High-resolution, high signal-to-noise optical spectra of the two program stars were taken in the framework of our ongoing
Table 6. The total extinction $A_{V}$ and the bolometric luminosity $L$ of the program stars.

\begin{tabular}{lrr}
\hline \hline IRAS & $A_{V}$ & $L$ at $D=5 \mathrm{kpc}$ \\
& $\mathrm{mag}$ & $L_{\odot}$ \\
\hline $08281-4850$ & 4.33 & 2450 \\
$14325-6428$ & 3.32 & 7250 \\
\hline
\end{tabular}

Table 7. Log of the high-resolution observations. For IRAS 082814850, a spectral gap occurs between $493 \mathrm{~nm}$ and $500 \mathrm{~nm}$. For IRAS 14325-6428, spectral gaps occur between $577 \mathrm{~nm}$ and $583 \mathrm{~nm}$ and between $854.4 \mathrm{~nm}$ and $864.5 \mathrm{~nm}$ due to the spatial gap between the two UVES CCDs.

\begin{tabular}{ccccc}
\hline \hline date & $\begin{array}{c}\text { UT } \\
\text { start }\end{array}$ & $\begin{array}{c}\text { exp.time } \\
(\mathrm{s})\end{array}$ & $\begin{array}{c}\text { wavelength } \\
\text { interval (nm) }\end{array}$ & $S / N$ \\
\hline \multicolumn{5}{c}{ IRAS 08281-4850 (NTT + EMMI) } \\
\hline $2003-02-04$ & $01: 11$ & 9000 & $395-795$ & 75 \\
\hline \multicolumn{5}{c}{ IRAS 14325-6428 (VLT-UT2 + UVES) } \\
\hline $2004-05-13$ & $07: 13$ & 1800 & $374.5-498$ & 130 \\
$2004-05-13$ & $07: 13$ & 1800 & $670.5-1055$ & 170 \\
$2004-05-13$ & $06: 39$ & 1800 & $477.5-681$ & 190 \\
\hline
\end{tabular}

program to study the photospheric chemical composition of stars in their last stages of evolution (e.g. Reyniers et al. 2004; Reyniers \& Van Winckel 2003; Van de Steene \& van Hoof 2003). IRAS 14325-6428 is observed with UVES on the VLT UT2 telescope (Kueyen), as a member of a larger sample of seven post-AGB objects that were observed in service mode during ESO period \#73. IRAS 08281-4850 was observed in visitor mode with the EMMI echelle grating \#9 with cross disperser $\# 3$ at the NTT during period \#70. The resolving power of the UVES spectra varies between $\sim 55000$ and $\sim 60000$. The resolving power of our EMMI spectra is significantly lower $(\sim 8400)$. The spectral interval covered and some other details about the observations are given in Table 7.

The reduction of our UVES spectra was performed in the dedicated "UVES context" of the MIDAS environment and included bias correction, cosmic hit correction, flat-fielding, background correction and sky correction. We used average extraction to convert frames from pixel-pixel to pixel-order space. The reduction of our EMMI spectrum was done within the echelle package in IRAF following the user's guide by Willmarth \& Barnes (1994). The spectra were normalised by dividing the individual orders by a smoothed spline function defined through interactively identified continuum points. For a detailed description of the reduction procedure, we refer to Reyniers (2002). In Table 7, we also list some indicative signal-to-noise values of the final data product.

Sample spectra of our programme stars can be found in Figs. 3 and 6. In Fig. 3, the spectra of IRAS 08281-4850 and IRAS 14325-6428 are compared with the spectra of the A7Iab standard HD 81471 and the non-enriched post-AGB star HD 133656. The spectrum of HD 81471 is retrieved from UVESPOP, the library of high-resolution UVES spectra of stars across the Hertzsprung-Russell diagram (Bagnulo et al. 2003). HD 133656 is discussed in Van Winckel et al. (1996). The spectrum of HD 133656 is an ESO1.5-m+FEROS spectrum taken on June 27, 2001. This object has atmospheric parameters comparable with the program stars $\left(T_{\text {eff }}, \log g, \xi_{\mathrm{t}}\right)=(8000 \mathrm{~K}, 1.0$, $\left.3.0 \mathrm{~km} \mathrm{~s}^{-1}\right)$ but is slightly more metal deficient $([\mathrm{Fe} / \mathrm{H}]=-0.9)$. The s-process enhancement of the two IRAS stars is clear from the Ba II line. IRAS 08281-4850 is the strongest s-enriched one, 

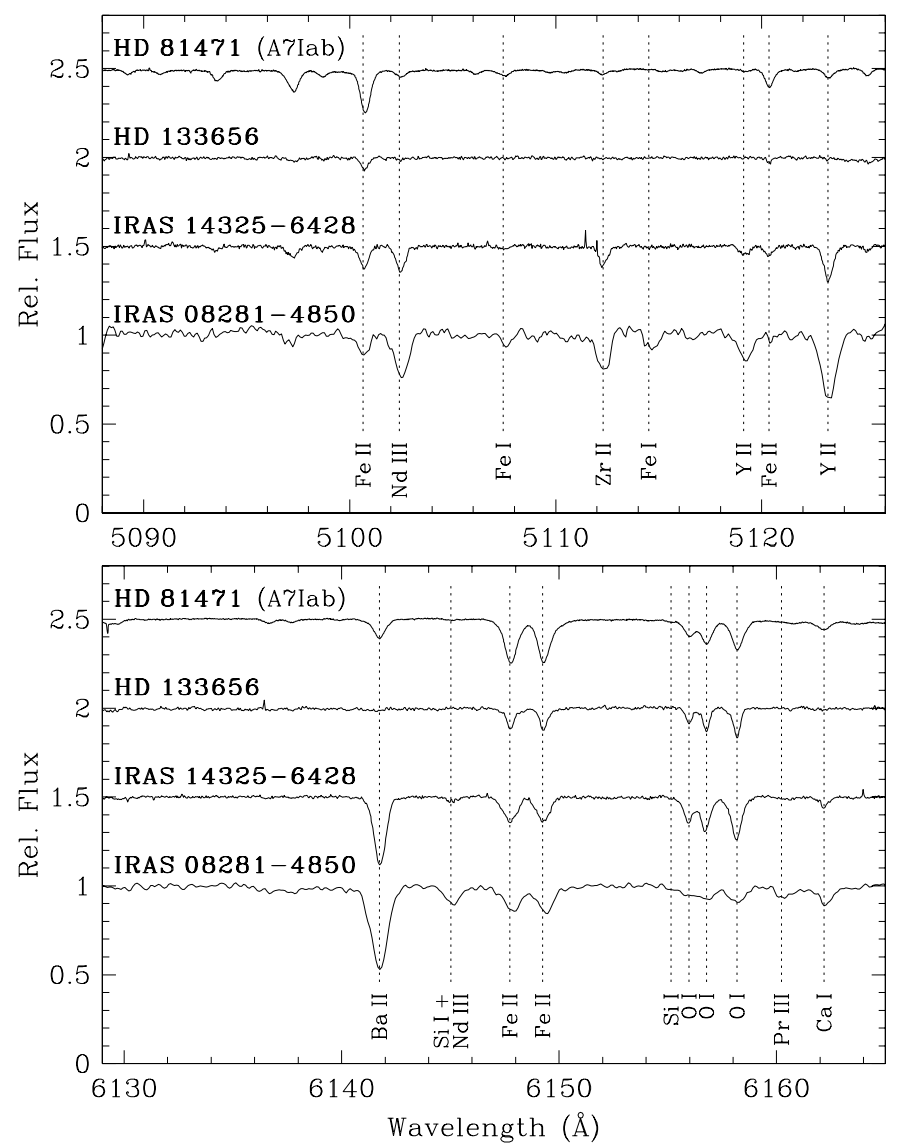

Fig. 3. The spectra of IRAS $14325-6428$ and IRAS 08281-4850 compared with the A7Iab standard HD 81471 and the non-enriched postAGB star HD 133656.

but the stronger lines are also due to a slightly lower temperature of this object.

\section{Abundance analysis and results}

\subsection{Method}

The general methodology is already extensively discussed in our previous papers (e.g. Reyniers et al. 2004; Deroo et al. 2005) and will not be repeated here. Here we only remind that we make use of the latest Atlas models (Castelli \& Kurucz 2004) in combination with the latest version (April 2002) of Sneden's LTE line analysis program MOOG (Sneden 1973). The value of the microturbulent velocity of IRAS 14325-6428 derived from the Fe II lines is high $\left(\xi_{\mathrm{t}}=10 \mathrm{~km} \mathrm{~s}^{-1}\right)$, but this high value is also supported by the results of other ions as can be seen in Fig. 4 .

Since the spectral resolution of the UVES spectra is much higher than that of the EMMI spectrum, we started the analysis with IRAS 14325-6428. In order to avoid undetected blends that are caused by the lower spectral resolution of the EMMI spectrum, we took the line list of IRAS $14325-6428$ as our starting point for the analysis of IRAS 08281-4850. As a consequence, all lines that we used for IRAS 08281-4850 are also present in the line list of IRAS 14325-6428. This choice ensures the consistency of the analyses, implying that a comparison between the abundance results is highly reliable. Due to the stronger s-process enrichment of IRAS 08281-4850 compared to IRAS 14325-6428, the spectrum of IRAS 08281-4850 shows significantly more lines of s-process elements. Therefore,

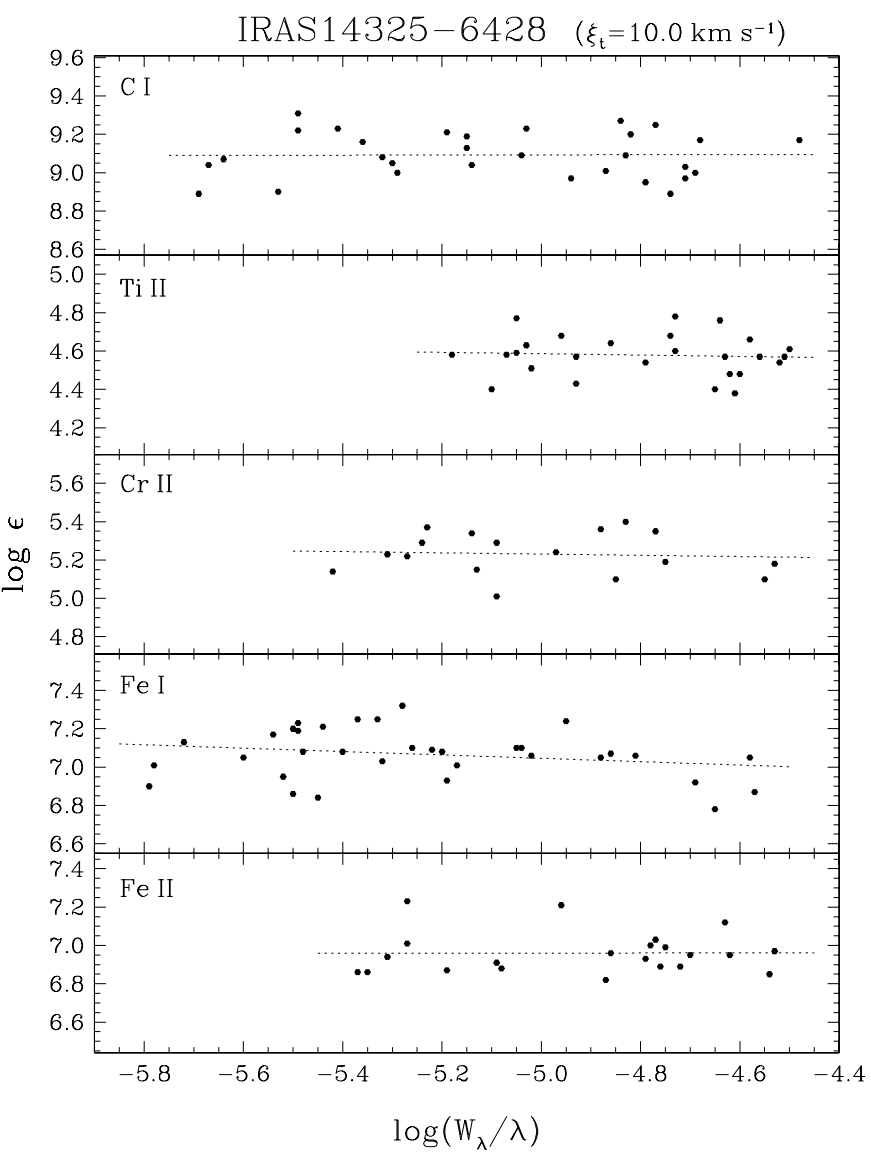

Fig. 4. The relatively high microturbulent velocity $\left(\xi_{\mathrm{t}}=10 \mathrm{~km} \mathrm{~s}^{-1}\right)$ for IRAS $14325-6428$ is also supported by the results of ions other than Fe II.

we performed an extra search for s-process lines in the spectrum of IRAS 08281-4850. This explains why we have used 12 La II lines and $1 \mathrm{Nd}$ II in the analysis of IRAS 08281-4850, against only 5 La II lines and no Nd II lines for IRAS 14325-6428.

\subsection{Abundance results}

The final results of our stellar atmosphere parameter determination and our abundance analysis are listed in Table 8. The first column of this table gives the actual ion. Then, for each star, the following columns are listed: the number of lines used; the mean equivalent width in $\mathrm{m} \AA$; the absolute abundances by number $\log \epsilon=\log (N(\mathrm{el}) / N(\mathrm{H}))+12$; the line-to-line scatter $\sigma_{\mathrm{lt}}$; the abundance relative to iron $[\mathrm{el} / \mathrm{Fe}]$, and an estimate of the total uncertainty on the abundance $\sigma_{\text {tot }}$ (see Sect. 4.3). For the references of the solar abundances (the middle column) needed to calculate the [el/Fe] values: see Reyniers et al. (2007).

The abundances are also graphically presented in Fig. 5. On this figure, the different groups of elements are marked with different symbols. We will summarize the main results for each of these groups.

Metallicity Both stars are mildly metal deficient, with iron abundances of $[\mathrm{Fe} / \mathrm{H}]=-0.33$ and -0.55 for IRAS 082814850 and IRAS 14325-6428 respectively. The other iron peak elements, if present, follow this deficiency.

CNO-elements Both stars are clearly carbon enriched, with an enrichment around $[\mathrm{C} / \mathrm{Fe}] \simeq+1$ for both stars. As a 
Table 8. Abundance results for IRAS 08281-4850 and IRAS14325-6428. For the explanation of the columns: see Sect. 4.2.

\begin{tabular}{|c|c|c|c|c|c|c|c|c|c|c|c|c|c|}
\hline \multicolumn{8}{|c|}{$\begin{array}{c}\text { IRAS } 08281-4850 \\
T_{\text {eff }}=7750 \mathrm{~K} \\
\log g=1.0(\mathrm{cgs}) \\
\xi_{\mathrm{t}}=4.5 \mathrm{~km} \mathrm{~s}^{-1}\end{array}$} & \multicolumn{6}{|c|}{$\begin{aligned} \text { IRAS } & \mathbf{1 4 3 2 5}-\mathbf{6 4 2 8} \\
T_{\mathrm{eff}} & =8000 \mathrm{~K} \\
\log g & =1.0(\mathrm{cgs}) \\
\xi_{\mathrm{t}} & =10.0 \mathrm{~km} \mathrm{~s}^{-1}\end{aligned}$} \\
\hline ion & $N$ & $\overline{W_{\lambda}}$ & $\log \epsilon$ & $\sigma_{\mathrm{ltl}}$ & {$[\mathrm{el} / \mathrm{Fe}]$} & $\sigma_{\text {tot }}$ & sun & $N$ & $\overline{W_{\lambda}}$ & $\log \epsilon$ & $\sigma_{\mathrm{ltl}}$ & {$[\mathrm{el} / \mathrm{Fe}]$} & $\sigma_{\text {tot }}$ \\
\hline C I & 12 & 117 & 9.17 & 0.24 & 0.93 & 0.15 & 8.57 & 30 & 64 & 9.09 & 0.12 & 1.07 & 0.13 \\
\hline $\mathrm{N}_{\mathrm{I}}$ & 2 & 87 & 8.21 & 0.14 & 0.55 & 0.20 & 7.99 & 6 & 94 & 7.98 & 0.18 & 0.54 & 0.14 \\
\hline O I & 2 & 84 & 8.89 & 0.10 & 0.36 & 0.22 & 8.86 & 10 & 72 & 8.89 & 0.11 & 0.58 & 0.14 \\
\hline $\mathrm{NaI}$ & 1 & 61 & 6.76 & & 0.76 & 0.25 & 6.33 & 2 & 16 & 6.50 & 0.02 & 0.72 & 0.20 \\
\hline Mg I & 1 & 107 & 7.31 & & 0.10 & 0.24 & 7.54 & 3 & 72 & 7.52 & 0.13 & 0.53 & 0.18 \\
\hline Mg II & & & & & & & 7.54 & 3 & 56 & 7.25 & 0.09 & 0.26 & 0.19 \\
\hline $\mathrm{Al} \mathrm{I}$ & & & & & & & 6.47 & 1 & 147 & 5.74 & & -0.18 & 0.23 \\
\hline$S_{\text {I }}$ & & & & & & & 7.33 & 1 & 17 & 7.26 & & 0.48 & 0.24 \\
\hline $\mathrm{Ca} I$ & 2 & 72 & 6.40 & 0.08 & 0.37 & 0.20 & 6.36 & 2 & 18 & 6.20 & 0.02 & 0.39 & 0.20 \\
\hline Sc II & 3 & 110 & 3.25 & 0.11 & 0.41 & 0.12 & 3.17 & 4 & 55 & 3.01 & 0.07 & 0.39 & 0.11 \\
\hline Ti II & 9 & 113 & 4.81 & 0.15 & 0.12 & 0.07 & 5.02 & 26 & 84 & 4.58 & 0.11 & 0.11 & 0.04 \\
\hline $\mathrm{CrI}$ & 1 & 122 & 5.60 & & 0.26 & 0.22 & 5.67 & 2 & 61 & 5.34 & 0.10 & 0.22 & 0.16 \\
\hline Cr II & 9 & 70 & 5.40 & 0.26 & 0.06 & 0.10 & 5.67 & 17 & 58 & 5.23 & 0.11 & 0.11 & 0.04 \\
\hline Mn II & & & & & & & 5.39 & 2 & 24 & 5.07 & 0.11 & 0.23 & 0.15 \\
\hline $\mathrm{Fe} I$ & 19 & 79 & 7.29 & 0.14 & 0.11 & 0.11 & 7.51 & 34 & 37 & 7.07 & 0.13 & 0.11 & 0.11 \\
\hline Fe II & 15 & 98 & 7.18 & 0.11 & 0.00 & & 7.51 & 22 & 74 & 6.96 & 0.11 & 0.00 & \\
\hline Ni II & & & & & & & 6.25 & 3 & 33 & 5.67 & 0.24 & -0.03 & 0.12 \\
\hline Y II & 9 & 116 & 3.81 & 0.17 & 1.90 & 0.08 & 2.24 & 15 & 70 & 3 & 0.19 & 1.32 & 0.06 \\
\hline Zr II & 1 & 170 & 3.85 & & 1.58 & 0.26 & 2.60 & 14 & 91 & 3.19 & 0.14 & 1.14 & 0.06 \\
\hline $\mathrm{Ba}$ II & & & & & & & 2.13 & 1 & 84 & 3.13 & & 1.55 & 0.20 \\
\hline La II & 12 & 75 & 2.93 & 0.18 & 2.13 & 0.08 & 1.13 & 5 & 54 & 1.89 & 0.10 & 1.31 & 0.06 \\
\hline Ce II & & & & & & & 1.58 & 2 & 16 & 2.15 & 0.05 & 1.12 & 0.15 \\
\hline Nd II & 1 & 52 & 2.78 & & 1.61 & 0.21 & 1.50 & & & & & & \\
\hline Nd III & 1 & 101 & 2.57 & & 1.40 & 0.22 & 1.50 & 3 & 60 & 2.00 & 0.11 & 1.05 & 0.14 \\
\hline \multirow[t]{8}{*}{ Sm II } & & & & & & & 1.01 & 1 & 11 & 1.69 & & 1.23 & 0.20 \\
\hline & \multicolumn{7}{|c|}{ summary } & \multicolumn{6}{|c|}{ summary } \\
\hline & \multicolumn{7}{|c|}{$[\mathrm{Fe} / \mathrm{H}]=-0.33$} & \multicolumn{6}{|c|}{$[\mathrm{Fe} / \mathrm{H}]=-0.55$} \\
\hline & \multicolumn{7}{|c|}{$\mathrm{C} / \mathrm{O}=1.9$} & \multicolumn{6}{|c|}{$\mathrm{C} / \mathrm{O}=1.6$} \\
\hline & \multicolumn{7}{|c|}{$[\alpha / \mathrm{Fe}]=+0.2(\alpha: \mathrm{Mg}, \mathrm{Ca}, \mathrm{Ti})$} & \multicolumn{6}{|c|}{$[\alpha / \mathrm{Fe}]=+0.3(\alpha: \mathrm{Mg}, \mathrm{S}, \mathrm{Ca}, \mathrm{Ti})$} \\
\hline & \multicolumn{7}{|c|}{$[\mathrm{l} / \mathrm{Fe}]=+1.74$} & \multicolumn{6}{|c|}{$[1 \mathrm{~s} / \mathrm{Fe}]=+1.23$} \\
\hline & \multirow{2}{*}{\multicolumn{7}{|c|}{$[\mathrm{hs} / \mathrm{Fe}]=+1.91$}} & \multicolumn{6}{|c|}{$[\mathrm{hs} / \mathrm{Fe}]=+1.29$} \\
\hline & & & & \multicolumn{4}{|c|}{$[\mathrm{hs} / \mathrm{ls}]=+0.17$} & \multicolumn{6}{|c|}{$[\mathrm{hs} / \mathrm{ls}]=+0.06$} \\
\hline
\end{tabular}

consequence, we derive also high $\mathrm{C} / \mathrm{O}$ number ratios for both stars. One has to note, however, that in IRAS 08281-4850 the uncertainty on the oxygen abundance prevents an accurate $\mathrm{C} / \mathrm{O}$ number ratio for this star.

$\alpha$-elements The simple mean of the $[\mathrm{el} / \mathrm{Fe}]$ values of the (available) $\alpha$-elements yields $[\alpha / \mathrm{Fe}]=+0.2$ and +0.3 for IRAS 08281-4850 and IRAS 14325-6428 respectively. Such an enhancement is normal for stars in this metallicity range, as a consequence of the galactic chemical evolution and therefore does not correspond to an intrinsic enhancement.

s-process elements It is clear that the s-process enrichment of the two objects under study is very strong.

The s-process elements observed in evolved stars can be divided into two groups: the light s-process elements around the magic neutron number 50 ( $\mathrm{Sr}, \mathrm{Y}, \mathrm{Zr}$ ) and the heavy s-process elements around the magic neutron number 82 (Ba, La, Ce, Pr, Nd, Sm). Three s-process indices are generally defined: [ls/Fe], [hs/Fe] and $[\mathrm{hs} / \mathrm{ls}$. To be consistent with our earlier papers on similar stars (Van Winckel \& Reyniers 2000; Reyniers et al. 2004; Reyniers \& Cuypers 2005), we define the ls-index as the mean of the $\mathrm{Y}$ and $\mathrm{Zr}$ abundances and the hs-index as the mean of the $\mathrm{Ba}, \mathrm{La}, \mathrm{Nd}$ and $\mathrm{Sm}$ abundances, with unavailable elements estimated using the tables of Malaney (1987). All indices are listed in Table 8.

\subsection{Uncertainty estimates}

For the error analysis, we followed the same method as described in Deroo et al. (2005). We slightly changed formula (1) of this paper, in the sense that for the uncertainty induced by the model $\sigma_{\text {mod }}$, we confined the parameter space to consistent models, i.e. models for which there is ionisation equilibrium between Fe I and Fe II. To be more precise, in order to calculate $\sigma_{\text {mod }}$, we studied the abundance changes for two different consistent models $\left(T_{\text {eff }}=7750 \mathrm{~K}, \log g=0.6\right)$ and $\left(T_{\text {eff }}=8250 \mathrm{~K}\right.$, $\log g=1.5)$, together with a change of the microturbulent velocity of $\xi_{t}=2 \mathrm{~km} \mathrm{~s}^{-1}$. The total uncertainty on the $[\mathrm{el} / \mathrm{Fe}]$ abundances $\sigma_{\text {tot }}$ can be found in Table 8 , and is the quadratic sum of the uncertainty on the mean due to line-to-line scatter, the uncertainty induced by the model, and the uncertainty on the $\mathrm{Fe}$ abundance:

$\sigma_{\mathrm{tot}}=\sqrt{\left(\frac{\sigma_{\mathrm{ltl}}}{\sqrt{\mathrm{N}_{\mathrm{el}}}}\right)^{2}+\left(\sigma_{\mathrm{mod}}\right)^{2}+\left(\frac{\sigma_{\mathrm{Fe}}}{\sqrt{\mathrm{N}_{\mathrm{Fe}}}}\right)^{2}}$

If less than 5 lines were available, a line-to-line scatter of $0.2 \mathrm{dex}$ was applied. 


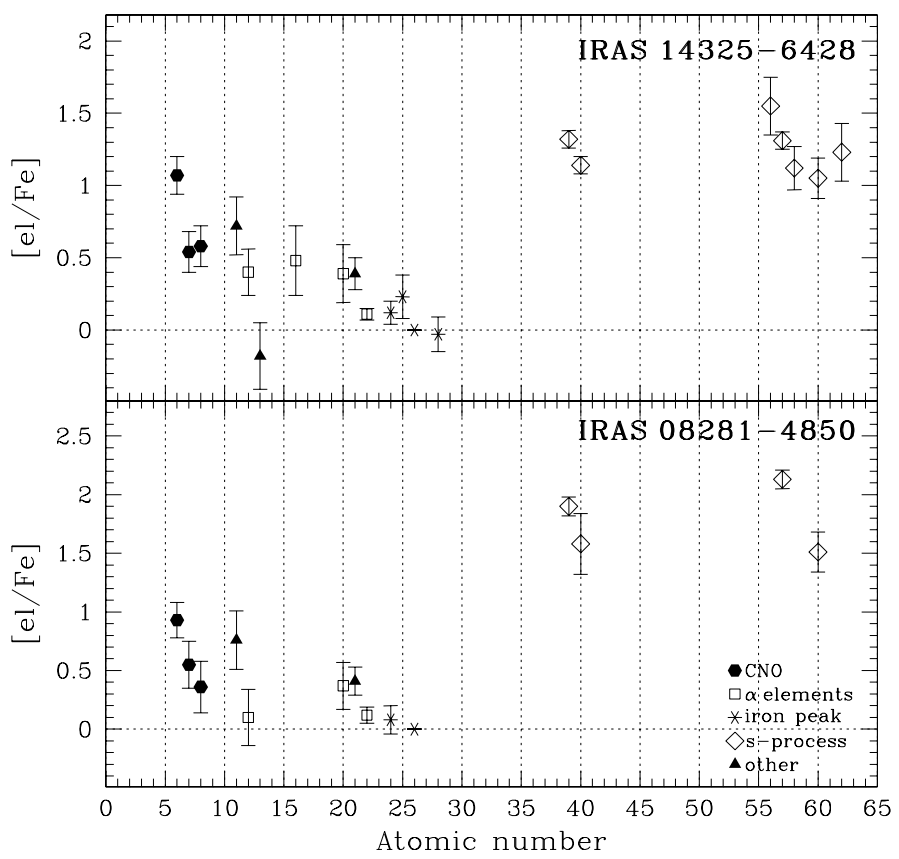

Fig. 5. [el/Fe] values for IRAS 14325-6428 (upper panel) and IRAS 08281-4850 (lower panel). The uncertainty on the [el/Fe] values is the total uncertainty $\sigma_{\text {tot }}$ as listed in Table 8 .

\section{Diffuse interstellar bands}

Diffuse Interstellar Bands (DIBs) are broad absorption lines of interstellar origin that are seen in the spectra of reddened objects. The carriers of these DIBs are still not known, but polycyclic aromatic hydrocarbons (PAHs) are amongst the most probable candidate carriers. Due to the severe mass loss in the preceding AGB phase, post-AGB stars are often enshrouded by carbon-rich circumstellar dust, causing severe reddening. Therefore, postAGB stars are ideal testlabs to search for possible circumstellar DIBs. If, on the other hand, the DIBs are detected to be interstellar, a rough division can be made between the interstellar and circumstellar component of the total reddening towards the post-AGB star, since some DIBs correlate quite well with the interstellar reddening.

\subsection{DIBs in IRAS 14325-6428}

During our analysis of IRAS 14325-6428, we realised that strong DIBs are indeed present in this spectrum. We initiated a systematic search for DIBs. First, we selected eight well-known and widely studied DIBs from the list in Herbig (1995). For each of these DIBs, we made a spectral synthesis in the vicinity of the DIB wavelength, based on the abundances found in our abundance analysis (Table 8). The DIB can then easily be identified as the spectral feature that is not fitted. Four DIBs are shown in Fig. 6. The studied DIBs in the spectrum of IRAS 143256428 are listed in Table 9. The columns of this table represent: rest wavelength of the DIB; the observed wavelength in IRAS 14325-6428; the heliocentric radial velocity of the DIB; the measured equivalent width; the intrinsic strength of the DIB as seen in the DIB standard HD 183143; and the (interstellar) reddening derived from the latter relation. The reference for Col. (1) and (5) is Herbig (1995).

The heliocentric radial velocities of the DIBs show some scatter, but they are definitely different from the heliocentric velocity of the star $\left(-87 \mathrm{~km} \mathrm{~s}^{-1}\right)$ and show velocities very different

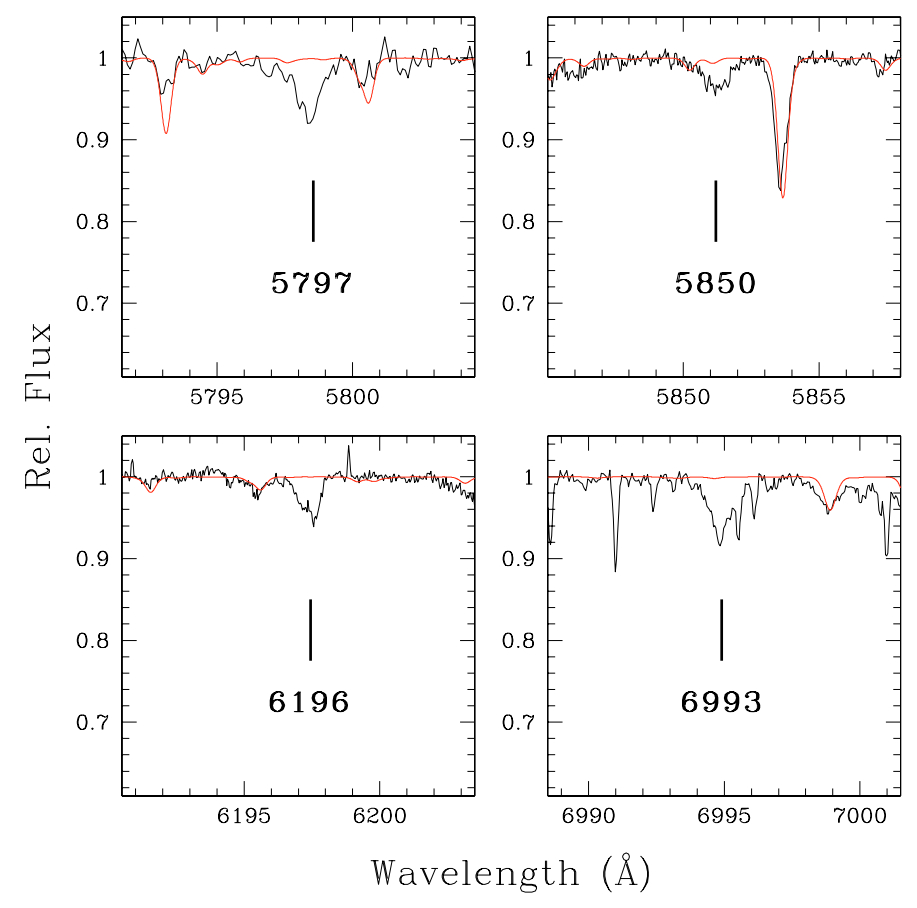

Fig. 6. Four clear diffuse interstellar bands in the spectrum of IRAS 14325-6428. A spectral synthesis is overplotted in red, to facilitate the DIB-detection.

from any expected outflow of the circumstellar material. Therefore, the DIBs seen in the spectrum of IRAS 14325-6428 are the imprint of an interstellar cloud between the object and the observer. A rough estimate for the distance towards the DIB producing cloud is obtained through the formula of Lang (1980), which describes the rotation of the Galactic Plane, yielding $1.6 \mathrm{kpc}$. Obviously, this distance is also a lower limit for the distance towards IRAS 14325-6428. The scatter in the DIB velocities could be caused by different DIB producing clouds in the line of sight towards IRAS 14325-6428, but also the rest wavelengths of the DIBs are difficult to quantify due to their complex fine structure (e.g. Galazutdinov et al. 2003).

For most DIBs, there is a correlation between its strength and the reddening that is caused by the DIB producing cloud. The DIB standard that is often used to quantify this relation, is HD $183143(E(B-V)=1.28)$. In Table 9, the DIB strengths of this standard, normalized to $E(B-V)=1$, are given in Col. 5, together with the inferred reddening for the DIB producing cloud towards IRAS 14325-6428 (Col. 6). A mean interstellar reddening of $E(B-V)=0.6$ is obtained. If we combine this with the total reddening towards IRAS $14325-6428$ of $E(B-V)_{\text {tot }}=1.1$ (Table 6), we can conclude that a significant fraction (around $50 \%$ ) of the total reddening towards IRAS $14325-6428$ is of interstellar origin.

\subsection{DIBs in IRAS $08281-4850$}

Due to the lower resolution and the lower $\mathrm{S} / \mathrm{N}$ ratio of the IRAS 08281-4850 spectra, we were not able to do a similar study for this source. A qualitative comparison of the strength of the strong DIB at $6196 \AA$ indicates that IRAS $08281-4850$ has certainly weaker DIBs than IRAS 14325-6428. The total reddening towards IRAS 08281-4850 is, however, stronger than the one towards IRAS 14325-6428, implying that the circumstellar 
Table 9. Studied DIBs in the spectrum of IRAS 14325-6428.

\begin{tabular}{rrrrrr}
\hline \hline $\begin{array}{r}\mathrm{DIB} \lambda \\
(\AA)\end{array}$ & $\begin{array}{r}\text { obs. } \lambda \\
(\AA)\end{array}$ & $\begin{array}{r}V_{\text {helio }} \\
\left(\mathrm{km} \mathrm{s}^{-1}\right)\end{array}$ & $\begin{array}{r}E W \\
(\AA)\end{array}$ & $\begin{array}{r}\frac{E W}{E(B-V)} \\
\text { HD 183143 }\end{array}$ & $\begin{array}{r}E(B-V) \\
\text { from DIB }\end{array}$ \\
\hline 5780.45 & 5779.43 & -16.6 & 0.417 & 0.626 & 0.7 \\
5796.98 & 5796.15 & -6.3 & 0.087 & 0.186 & 0.5 \\
5849.65 & 5849.57 & -1.1 & 0.042 & 0.064 & 0.7 \\
6195.95 & 6195.61 & -13.2 & 0.043 & 0.063 & 0.7 \\
6283.86 & 6283.60 & -9.2 & 1.043 & 1.520 & 0.7 \\
6379.20 & 6378.95 & -8.5 & 0.041 & 0.096 & 0.4 \\
6613.62 & 6613.28 & -12.4 & 0.138 & 0.280 & 0.5 \\
6993.07 & 6992.82 & -7.7 & 0.079 & 0.142 & 0.6 \\
\hline mean $( \pm \sigma)$ & $-9.4( \pm 4.8)$ & & & $0.6( \pm 0.1)$ \\
\hline
\end{tabular}

component of the total reddening of IRAS 08281-4850 is probably large.

\section{Discussion}

IRAS 08281-4850 and IRAS 14325-6428 were selected on the basis of their position in the IRAS colour-colour diagram (Pottasch et al. 1988) and the lack of free-free radio continuum emission (Van de Steene \& Pottasch 1993, 1995). Both objects remained poorly studied until now. Our chemical analysis presented in this paper shows that both objects are among the hottest members of the s-process enhanced post-AGB stars known to date and illustrates that the rich IRAS legacy of objects in the transition between the AGB and the PN phase is far from being harvested. Although the distance to the objects is not well constrained, both objects do not show an extremely high luminosity (Table 6) and, given the sub-solar metallicity, both objects represent the final evolutionary phase of a star with a low initial $\operatorname{mass}\left(M_{\mathrm{i}}<2 M_{\odot}\right)$.

There is general agreement that the source of the neutrons for the s-process in low and intermediate AGB stars is the ${ }^{~}{ }^{13} \mathrm{C}$ source". A long standing problem is the formation of the ${ }^{13} \mathrm{C}$ pocket itself. Stellar models using a standard treatment of mixing cannot reproduce the ${ }^{13} \mathrm{C}$ pocket at a level which is high enough for the s-process to take place (Herwig 2005). Until now, there is no satisfying description for this phenomenon, and the different modeling groups use different descriptions. Many models use some kind of overshoot mechanism (e.g. Herwig et al. 1997). Also differential rotation has recently been studied as a possible mixing mechanism driving the proton engulfment, but this rotationally induced mixing alone cannot account for the formation of a large enough ${ }^{13} \mathrm{C}$ pocket (Herwig et al. 2003). Other groups avoid this formation problem by assuming an ad-hoc ${ }^{13} \mathrm{C}$ pocket in the He intershell (models by e.g. Gallino et al. 1998) or an adhoc proton density profile (models by e.g. Goriely \& Mowlavi 2000). The observed spread in s-process efficiency (see Sect. 1) is then reproduced by a variable ${ }^{13} \mathrm{C}$ pocket strength. Since the ${ }^{13} \mathrm{C}$ pocket is build upon primary synthesised ${ }^{12} \mathrm{C}$, the formation is thought to be largely independent of the initial metallicity and a richer s-process nucleosynthesis is expected for lower metallicity stars, because more neutrons become available per iron-seed. In practise, such a trend has not been found for post-AGB stars.

The very recent results of the population synthesis models by Bonačić Marinović et al. (2006) and Bonačić Marinović et al. (2007) are very interesting in this context. Their approach is to combine stellar population synthesis with a rapid stellar evolution code including AGB nucleosynthesis and evolution. Interestingly, their models do reproduce both the observed dichotomy (see Fig. 1d in Bonačić Marinović et al. 2006), and the observed spread. Contrary to previous models, only a limited spread in the strength of the ${ }^{13} \mathrm{C}$ pocket is needed to reproduce the observed efficiency spread. With the new results presented in this paper, we are able to add two important datapoints to constrain this promising new generation of models. Indeed, the two stars discussed here show a strong s-process enrichment, although they are only mildly metal deficient. Particularly the results of IRAS 08281-4850 are on the predicted boundaries of both the [hs/ls] index (Fig. 3 of Bonačić Marinović et al. 2007) and the $\mathrm{Zr}$ overabundance (Fig. 8 of Bonačić Marinović et al. 2007).

\section{Conclusion}

With the comprehensive abundance analysis presented in this paper, the objects IRAS 08281-4850 and IRAS 14325-6428 join the group of post-AGB stars with a clear post third dredge-up signature, since they do not only show a clear carbon enhancement, but also a strong enrichment in s-process elements. This enrichment is surprisingly strong with a high ratio of heavy versus light s-process elements, despite the only mild metal deficiency of both objects. This provides additional evidence of the intrinsic s-process efficiency spread in (post-)AGB stars. A systematic analysis of post-AGB stars in the PNe locus of the IRAS colour-colour diagram would be a very rewarding program to study the relation between the AGB nucleosynthesis, the dredgeup efficiency and the overall stellar evolution of the central star.

Acknowledgements. MR is grateful to Sara Regibo for the preliminary analysis of IRAS 14325-6428 in an early stage of the paper. The Geneva staff is thanked for observation time on the Euler telescope. MR acknowledges financial support from the Fund for Scientific Research - Flanders (Belgium). PvH acknowledges support from the Belgian Science Policy Office through grant MO/33/017.

\section{References}

Bagnulo, S., Jehin, E., Ledoux, C., et al. 2003, The Messenger, 114, 10 Beichmann, C. A. 1985, Infrared Astronomical Satellite (IRAS) catalogs and atlases. Explanatory supplement, Pasadena: Jet Propulsion Laboratory (Beichmann, C.A.)

Bonačić Marinović, A., Izzard, R. G., Lugaro, M., \& Pols, O. R. 2006, Mem. Soc. Astron. Ital. 77, 879

Bonačić Marinović, A. A., Izzard, R. G., Lugaro, M., \& Pols, O. R. 2007, [arXiv: astro-ph/0703122]

Castelli, F., \& Kurucz, R. L. 2004 [arXiv: astro-ph/0405087]

Cohen, M., Wheaton, W. A., \& Megeath, S. T. 2003, AJ, 126, 1090

Deroo, P., Reyniers, M., Van Winckel, H., Goriely, S., \& Siess, L. 2005, A\&A, 438, 987

Egan, M. P., Price, S. D., Kraemer, K. E., et al. 2003, VizieR Online Data Catalog, 5114, 0

Epchtein, N., de Batz, B., Copet, E., et al. 1994, Ap\&SS, 217, 3

Fitzpatrick, E. L. 1999, PASP, 111, 63

Fouqué, P., Chevallier, L., Cohen, M., et al. 2000, A\&AS, 141, 313

Galazutdinov, G. A., Musaev, F. A., Bondar, A. V., \& Krełowski, J. 2003, MNRAS, 345, 365

Gallino, R., Arlandini, C., Busso, M., et al. 1998, ApJ, 497, 388

Garcia-Lario, P., Manchado, A., Pych, W., \& Pottasch, S. R. 1997, A\&AS, 126, 479 (GL97)

Goriely, S., \& Mowlavi, N. 2000, A\&A, 362, 599

Harmanec, P., \& Božić, H. 2001, A\&A, 369, 1140

Herbig, G. H. 1995, ARA\&A, 33, 19

Herwig, F. 2005, ARA\&A, 43, 435

Herwig, F., Bloecker, T., Schoenberner, D., \& El Eid, M. 1997, A\&A, 324, L81

Herwig, F., Langer, N., \& Lugaro, M. 2003, ApJ, 593, 1056

Høg, E., Fabricius, C., Makarov, V. V., et al. 2000, A\&A, 355, L27

Johnson, H. L. 1965, Communications of the Lunar and Planetary Laboratory, 3, 73

Johnson, H. L. 1966, ARA\&A, 4, 193

Kent, S. M. 1985, PASP, 97, 165

Lamla, E. 1982, in Landolt-Bornstein Series, vol. 2b, Stars and Star Clusters ed. K. Schaifers, \& H.H. Voigt (Berlin: Springer Verlag) 
Lang, K. R. 1980, Astrophysical Formulae, A Compendium for the Physicist and Astrophysicist, XXIX, 783 pp. 46 figs., 69 tabs.. (Berlin, Heidelberg, New York: Springer-Verlag, Also Springer Study Edition)

Maas, T., Van Winckel, H., \& Lloyd Evans, T. 2005, A\&A, 429, 297

Malaney, R. A. 1987, Ap\&SS, 137, 251

Perryman, M. A. C., Lindegren, L., Kovalevsky, J., et al. 1997, A\&A, 323, L49 Pottasch, S. R., Olling, R., Bignell, C., \& Zijlstra, A. A. 1988, A\&A, 205, 248 Reyniers, M. 2002, Ph.D. Thesis, K.U.Leuven

Reyniers, M., \& Van Winckel, H. 2003, A\&A, 408, L33

Reyniers, M., \& Cuypers, J. 2005, A\&A, 432, 595

Reyniers, M., Van Winckel, H., Gallino, R., \& Straniero, O. 2004, A\&A, 417, 269

Reyniers, M., Abia, C., Van Winckel, H., et al. 2007, A\&A, 461, 641

Rufener, F., \& Nicolet, B. 1988, A\&A, 206, 357

Skrutskie, M. F., Schneider, S. E., Stiening, R., et al. 1997, in ASSL 210: The Impact of Large Scale Near-IR Sky Surveys, ed. F. Garzon, N. Epchtein, A. Omont, B. Burton, \& P. Persi, 25
Sneden, C. A. 1973, Ph.D. Thesis, University of Texas at Austin

Straniero, O., Domínguez, I., Cristallo, R., \& Gallino, R. 2003, Publ. Astron. Soc. Austral., 20, 389

Thomas, J. A., Hyland, A. R., \& Robinson, G. 1973, MNRAS, 165, 201

Van de Steene, G. C. M., \& Pottasch, S. R. 1993, A\&A, 274, 895

Van de Steene, G. C., \& Pottasch, S. R. 1995, A\&A, 299, 238

Van de Steene, G. C., \& van Hoof, P. A. M. 2003, A\&A, 406, 773

Van de Steene, G. C., van Hoof, P. A. M., \& Wood, P. R. 2000, A\&A, 362, 984 (VdSO0)

van Hoof, P. A. M., Oudmaijer, R. D., \& Waters, L. B. F. M. 1997, MNRAS, 289,371

Van Winckel, H. 2003, ARA\&A, 41, 391

Van Winckel, H., \& Reyniers, M. 2000, A\&A, 354, 135

Van Winckel, H., \& Reyniers, M. 2001, in Post-AGB Objects as a Phase of Stellar Evolution, ed. R. Szczerba, \& S. K. Górny, 257

Van Winckel, H., Oudmaijer, R. D., \& Trams, N. R. 1996, A\&A, 312, 553 


\section{Online Material}


M. Reyniers et al.: Abundance analysis of IRAS 08281-4850 and IRAS 14325-6428, Online Material p 2

Table 4. Absolute flux calibration for the various photometric bands used in this paper.

\begin{tabular}{lcc}
\hline \hline phot. band & $\begin{array}{c}0 \text {-mag flux } \\
\mathrm{W} \mathrm{m}^{-2} \mu \mathrm{m}^{-1}\end{array}$ & reference \\
& $3.129 \times 10^{-9}$ & Cohen et al. (2003) \\
2MASS $J$ & $1.133 \times 10^{-9}$ & $"$ \\
2MASS $H$ & $4.283 \times 10^{-10}$ & $"$ \\
2MASS $K_{\mathrm{s}}$ & $1.196 \times 10^{-8}$ & Lamla (1982) \\
Cousins $I_{\mathrm{C}}$ & $1.20 \times 10^{-8}$ & Fouqué et al. (2000) \\
DENIS Gunn- $i$ &, \\
DENIS $J$ & $3.17 \times 10^{-9}$ & $"$ \\
DENIS $K_{\mathrm{s}}$ & $4.34 \times 10^{-10}$ &, \\
Geneva $U$ & $5.754 \times 10^{-8}$ & Rufener \& Nicolet (1988) \\
Geneva $B$ & $2.884 \times 10^{-8}$ & $"$ \\
Geneva $V$ & $3.736 \times 10^{-8}$ & $"$ \\
Johnson $B$ & $7.20 \times 10^{-8}$ & Johnson (1965, 1966) \\
Johnson $V$ & $3.92 \times 10^{-8}$ & $"$ \\
Johnson $R$ & $1.76 \times 10^{-8}$ & $"$ \\
Johnson $J$ & $3.4 \times 10^{-9}$ & $"$ \\
Johnson $H$ & $1.26 \times 10^{-9}$ & $"$ \\
Johnson $K$ & $3.9 \times 10^{-10}$ & $"$ \\
MSSO $J$ & $3.03 \times 10^{-9}$ & Thomas et al. (1973) \\
MSSO $H$ & $1.17 \times 10^{-9}$ &, \\
MSSO $K$ & $4.02 \times 10^{-10}$ &, \\
MSSO $L$ & $6.18 \times 10^{-11}$ & \\
\hline
\end{tabular}

Table 5. The spectral energy distribution (sorted by wavelength) given as $\lambda F_{\lambda}$ in SI units.

\begin{tabular}{|c|c|c|c|c|}
\hline \multirow{2}{*}{$\begin{array}{r}\lambda \\
\mu \mathrm{m}\end{array}$} & \multicolumn{2}{|c|}{ 08281-4850 } & \multicolumn{2}{|c|}{$14325-6428$} \\
\hline & $\begin{array}{c}\lambda F_{\lambda} \\
\mathrm{W} \mathrm{m}^{-2}\end{array}$ & $\begin{array}{c}\Delta \lambda F_{\lambda} \\
\mathrm{W} \mathrm{m}^{-2}\end{array}$ & $\begin{array}{c}\lambda F_{\lambda} \\
\mathrm{W} \mathrm{m} \mathrm{m}^{-2}\end{array}$ & $\begin{array}{c}\Delta \lambda F_{\lambda} \\
\mathrm{W} \mathrm{m}^{-2}\end{array}$ \\
\hline 0.3464 & $1.50 \times 10^{-15}$ & $2.2 \times 10^{-16}$ & $2.94 \times 10^{-14}$ & $7.6 \times 10^{-15}$ \\
\hline 0.4227 & $1.52 \times 10^{-14}$ & $1.5 \times 10^{-15}$ & $1.79 \times 10^{-13}$ & $1.7 \times 10^{-14}$ \\
\hline 0.4442 & & & $3.11 \times 10^{-13}$ & $6.6 \times 10^{-14}$ \\
\hline 0.5488 & $4.74 \times 10^{-14}$ & $2.2 \times 10^{-15}$ & $3.60 \times 10^{-13}$ & $1.7 \times 10^{-14}$ \\
\hline 0.5537 & & & $3.41 \times 10^{-13}$ & $5.8 \times 10^{-14}$ \\
\hline 0.6938 & $1.10 \times 10^{-13}$ & $1.6 \times 10^{-14}$ & & \\
\hline 0.7886 & $1.24 \times 10^{-13}$ & $5.9 \times 10^{-15}$ & & \\
\hline 0.7910 & & & $6.38 \times 10^{-13}$ & $1.2 \times 10^{-14}$ \\
\hline 0.7910 & & & $7.22 \times 10^{-13}$ & $2.7 \times 10^{-14}$ \\
\hline 1.2280 & & & $7.47 \times 10^{-13}$ & $3.5 \times 10^{-14}$ \\
\hline 1.2280 & & & $8.12 \times 10^{-13}$ & $5.4 \times 10^{-14}$ \\
\hline 1.2350 & $2.22 \times 10^{-13}$ & $5.5 \times 10^{-15}$ & $7.62 \times 10^{-13}$ & $2.1 \times 10^{-14}$ \\
\hline 1.2500 & $2.27 \times 10^{-13}$ & $1.1 \times 10^{-14}$ & $7.42 \times 10^{-13}$ & $3.5 \times 10^{-14}$ \\
\hline 1.6200 & $1.83 \times 10^{-13}$ & $8.6 \times 10^{-15}$ & & \\
\hline 1.6500 & & & $5.78 \times 10^{-13}$ & $2.7 \times 10^{-14}$ \\
\hline 1.6620 & $1.68 \times 10^{-13}$ & $4.9 \times 10^{-15}$ & $5.38 \times 10^{-13}$ & $1.6 \times 10^{-14}$ \\
\hline 2.1450 & & & $3.37 \times 10^{-13}$ & $1.6 \times 10^{-14}$ \\
\hline 2.1450 & & & $3.90 \times 10^{-13}$ & $3.4 \times 10^{-14}$ \\
\hline 2.1590 & $1.08 \times 10^{-13}$ & $2.9 \times 10^{-15}$ & $3.36 \times 10^{-13}$ & $1.0 \times 10^{-14}$ \\
\hline 2.1900 & $1.04 \times 10^{-13}$ & $3.9 \times 10^{-15}$ & & \\
\hline 2.2000 & & & $3.18 \times 10^{-13}$ & $1.5 \times 10^{-14}$ \\
\hline 3.6000 & & & $1.09 \times 10^{-13}$ & $1.1 \times 10^{-14}$ \\
\hline 8.2800 & & & $2.59 \times 10^{-13}$ & $1.1 \times 10^{-14}$ \\
\hline 12.0000 & $5.57 \times 10^{-13}$ & $2.7 \times 10^{-14}$ & $8.27 \times 10^{-13}$ & $5.0 \times 10^{-14}$ \\
\hline 12.1300 & & & $8.27 \times 10^{-13}$ & $4.4 \times 10^{-14}$ \\
\hline 14.6500 & & & $1.45 \times 10^{-12}$ & $8.8 \times 10^{-14}$ \\
\hline 21.3400 & & & $2.48 \times 10^{-12}$ & $1.5 \times 10^{-13}$ \\
\hline 25.0000 & $1.18 \times 10^{-12}$ & $7.1 \times 10^{-14}$ & $3.67 \times 10^{-12}$ & $1.4 \times 10^{-13}$ \\
\hline 60.0000 & $1.81 \times 10^{-13}$ & $1.8 \times 10^{-14}$ & $8.54 \times 10^{-13}$ & $9.5 \times 10^{-14}$ \\
\hline
\end{tabular}

